\section{Southern African Student Surgical Society (SASSS) and University of Cape Town Division of Global Surgery Symposium: Developing a pipeline of surgical student researchers collaboratively}

To the Editor: Academic conferences collaboratively organised by students and academic staff can be used as a means of increasing interest in undergraduate surgical research, particularly because there is limited time and opportunity to engage with research in a meaningful way in medical school curricula. A study conducted at the University of Cape Town (UCT) in 2014 evaluated medical students' perceptions of research. It found that students perceived research to be important, had a positive attitude towards it, and wanted to become involved in research activities. Despite this, not many had been involved in voluntary research (11\% of preclinical students and $12 \%$ of clinical students). Some of the barriers cited were perceived lack of training, time and opportunities, and lack of awareness of available research opportunities. ${ }^{[1]}$ The authors concluded that active work must be done to create an environment that fosters passion in clinical research to reverse the worldwide decline of clinician and surgical scientists. ${ }^{[2,3]}$

On 10 - 11 October 2020, the Southern African Student Surgical Society (SASSS) and the UCT Division of Global Surgery hosted a symposium entitled 'Reimagining perioperative care in Africa'. In the weeks preceding the symposium, a research competition was held as a means of encouraging undergraduate researchers to display their work. Research has been a major component of the annual SASSS symposium since its inception in 2013. However, because of COVID-19 restrictions in 2020, typical in-person participation could not occur. Instead, a virtual conference was held, which included an online research poster competition.

The top 3 research topics were presented by student researchers on an Instagram live feed that delegates and judges were invited to attend. This provided an opportunity for the top 3 researchers to practise their oral presentation skills and for symposium delegates to engage with the research topics by asking questions. Owing to our partnerships with companies, high-quality prizes were awarded to the winners. Internal motivation should be targeted to stimulate research involvement. Therefore, the authors suggest that prizes can act as positive reinforcement for those already interested in research. However, additional efforts need to be made to promote research participation among research-naive students, such as the utilisation of digital structures, mentorship and submission of manuscripts with simple methodologies, e.g. case reports and commentaries. ${ }^{[3,4]}$

Despite the pandemic and international travel restrictions, SASSS and the UCT Division of Global Surgery hosted a successful collaborative conference, including numerous international delegates and speakers.

In conclusion, an annual collaborative conference such as the SASSS symposium that showcases advances in surgical care, while promoting research and researchers in surgery, is a definite way of inspiring the next generation of surgical scientists and academic surgeons. ${ }^{[2,3]}$ Medical student participation in research is associated with positive short- and long-term scientific productivity, improved knowledge of and interest in research. ${ }^{[5]}$ Therefore, collaborating with academic staff and creating opportunities for students to participate in research could motivate and inspire more students to pursue academic-clinician and surgeon-scientist careers.

Acknowledgements. We acknowledge the assistance of Prof. Salome Maswime, head of the Division of Global Surgery at UCT, and the SAMRC Mid-Career Scientist programme, which funds the Global Surgery Student Research Group.

\section{I Majiet}

Global Surgery Student Research Group, Division of Global Surgery, Department of Surgery, Faculty of Health Sciences, University of Cape Town, and Southern African Student Surgical Society, South Africa mjtmog005@myuct.ac.za

\section{S Lee}

Faculty of Medicine and Health Sciences, Stellenbosch University, Cape Town, and Southern African Student Surgical Society, South Africa

\section{Z Y He}

Faculty of Health Sciences, University of Cape Town, and Southern African Student Surgical Society, South Africa

\section{$S$ Verhage}

Faculty of Health Sciences, University of Cape Town, and Southern African Student Surgical Society, South Africa

\section{S Breedt}

Faculty of Medicine and Health Sciences, Stellenbosch University, Cape Town, and Southern African Student Surgical Society, South Africa

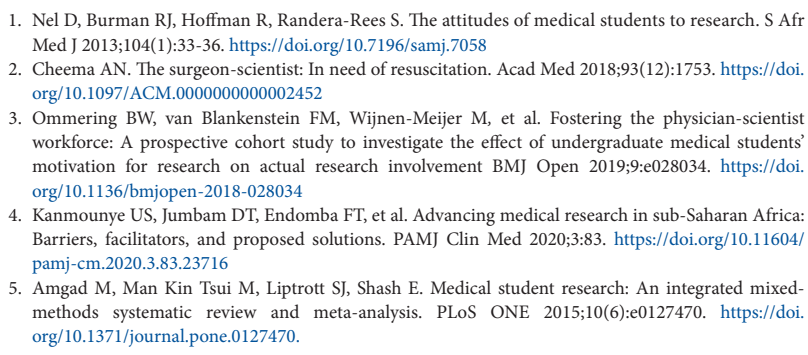
Med J 2013;104(1):33-36. https://doi.org/10.7196/samj.7058

2. Cheema AN. The surgeon-scientist: In need of resuscitation. Acad Med 2018;93(12):1753. https://doi. org/10.1097/ACM.0000000000002452

3. Ommering BW, van Blankenstein FM, Wijnen-Meijer M, et al. Fostering the physician-scientis workforce: A prospective cohort study to investigate the effect of undergraduate medical students motivation for research on actual research involvement BMJ Open 2019;9:e28034. https://doi org/10.1136/bmjopen-2018-028034

4. Kanmounye US, Jumbam DT, Endomba FT, et al. Advancing medical research in sub-Saharan Africe: Barriers, facilitators, and proposed solutions. PAMJ Clin Med 2020;3:83. https://doi.org/10.11604/ pamj-cm.2020.3.83.23716

5. Amgad M, Man Kin Tsui M, Liptrott SJ, Shash E. Medical student research: An integrated mixedmethods systematic review and meta-analysis. PLoS ONE 2015;10(6):e0127470. https://doi. org/10.1371/journal.pone.0127470.

S Afr Med J 2021;111(7):606. https://doi.org/10.7196/SAMJ.2021.v111i7.15684 